\title{
Impact of mosquito-borne diseases on global public health
}

\begin{abstract}
Mosquito-borne diseases are a concern of global public health and safety. Risk of mosquitoborne illness varies greatly with occupation, age, ethnicity, gender, income status, travel frequency, and climate change. Those at most risk of being infected by a mosquito-borne disease include frequent travelers, healthcare personnel, laboratory workers, and those whose occupation is mostly conducted outside or in wooded areas. Many people become sick after being bitten by an infected mosquito. Some people have mild short-term illness and in cases some people experience long-term illnesses. Severe cases of mosquito-borne diseases have resulted in death. Recommendations include investing in climate change, increasing the availability of vaccines globally, the use of health and mosquito surveillance systems, and forming regional and international action plans for disease control in the case of an endemic, epidemic, or a pandemic.
\end{abstract}

Volume 6 Issue I - 202I

\author{
Tina M. Penhollow,' Luis Torres² \\ 'Department of Exercise Science and Health Promotion, \\ Associate Professor, Florida Atlantic University College of \\ Science, USA \\ ${ }^{2}$ Department of Geosciences, Florida Atlantic University College \\ of Science, USA
}

\begin{abstract}
Correspondence: Tina M. Penhollow, Department of Exercise Science and Health Promotion, Associate Professor, Florida Atlantic University College of Science, USA, 777 Glades Road, FHI I-25B, Boca Raton, Florida 3343I, Tel 56 I2972643, Email tpenholl@fau.edu
\end{abstract}

Received: January 30, 202I | Published: February 10, 202 I

\section{Introduction}

Mosquitos are one of the most common groups of biting insects known worldwide. Mosquito-borne diseases are those spread by the bite of an infected mosquito. They are known to carry many diseases including malaria, Zika virus, dengue fever, yellow fever, chikungunya virus, West Nile virus, as well as Eastern Equine Encephalitis (EEE), Western Equine Encephalitis (WEE), and St. Louis Encephalitis (SLE). ${ }^{1}$ These diseases are known as mosquito-borne diseases due to their use of different genera of mosquitos as vectors. Mosquitos will typically carry pathogens in their bile, which is injected into a bitten individual in order to prevent the blood from coagulating. These diseases often put a very heavy toll on the body of an infected individual and may often be fatal. This is especially true in older individuals who are immune compromised and those without much access to medical care. According to a paper by Caraballo and King, ${ }^{2}$ mosquito-borne diseases cause an average of one million deaths on a global scale every year. A recent article stated that "mosquito transmitted infections post a significant threat to public health worldwide". ${ }^{3}$ The increase of the incidence of these diseases around the world is an important health issue that needs to be addressed.

According to the $\mathrm{CDC},{ }^{4}$ diseases from bites from vectors such as mosquitos, ticks, and fleas have more than tripled in the United States from 2004-2016. In 2017, an estimated 219 million new malaria cases occurred worldwide, with 435,000 deaths. The Zika virus epidemic infected more than one million people with thousands of infants with birth defects from 2015-2016. The yellow fever outbreak in Congo caused 7,334 suspected cases and 498 deaths. In 1999, the West Nile Virus quickly spread across the United States causing hundreds of deaths annually. Dengue fever is an occurring endemic in over 100 countries, consisting of around half of the world's population. Over one hundred million people are infected every year. ${ }^{5}$

The causes for the rise in cases in mosquito-borne diseases is generally thought to be a result of climate change. ${ }^{6}$ It is reported that climate change is likely affecting the amplitude of the El Nino/ southern oscillation climate pattern which affects precipitation patterns all over the world. ${ }^{7}$ Areas with higher temperatures will increase the transmission season as well as increase the rate at which diseases inside the mosquito vectors develop. ${ }^{8}$ This can be explained by certain pathogens becoming inert when reaching a certain temperature, leading to certain diseases becoming seasonal. Warmer overall temperatures also lead to new areas being accessible to these pathogens and allows the endemic populations of mosquitos to become vectors to these pathogens.

\section{Interventions}

Advances in technology and overall increases in living standards such as assess to clean water and vaccines among populations may also help to reduce the number of individuals who are infected by mosquito-borne diseases. ${ }^{9,10}$ Insecticides are often used in mosquito control, however, the overuse of them can cause just as many issues. For one, insecticides are often non-discriminatory in what they affect, killing many different species and having effects on many species that aren't particularly targeted. ${ }^{11}$ Moreover, the overuse of chemicals to control mosquito populations have resulted in a tolerance to the typical chemicals used, reducing their effectiveness.

There are also more experimental ways of reducing the infectivity of mosquito borne pathogens and reduce mosquito-borne disease incidence. The use of microbiota can shorten the lifespan of certain mosquito species in addition to blockage of pathogen transfer from the bites of mosquitos. ${ }^{12}$ Many mosquitos require the use of an environmentally microbiota within them in order to perform daily life activities such as growth and reproduction. In a study by Zheng et al. ${ }^{13}$ researchers were able to reduce the incidence rate of individuals who were bitten on and isolated island in Guang Zhou, China by $88.7-96.6 \%$ through releasing Wolbachia infected male mosquitos of the Aedes genus. These males would mate with normal females and due to the Wolbachia, their offspring would be unable develop into larvae due to cytoplasmic incompatibility. It is further discussed that the use of genetic engineering to the microbiota of these mosquitos 
could produce anti-pathogen effects, reducing the infectiveness of the mosquito-borne pathogens. These mosquitos could then breed with wild species and reduce the overall infectiveness of the disease pathogens of all interbred mosquitos. ${ }^{12}$

\section{Conclusion}

Mosquito-borne diseases have a significant impact on the health and economy of many populations worldwide. While populations in countries with less resources are more severely impacted, the recent change in climate and development have caused large increases in the incidence of these diseases in many regions. Perhaps more novel changes in technology, vector control, vaccine dissemination, pollution regulations, as well as environmental solutions may be the secret that allows countries of all levels of wealth to decrease the incidence of diseases being spread by mosquitos.

\section{Funding}

None.

\section{Acknowledgments}

None.

\section{Conflicts of interest}

The authors declare no conflicts of interest.

\section{References}

1. Centers for disease control and prevention (CDC). Mosquito-borne diseases. The National Institute for Occupational Safety and Health. 2021.
2. Caraballo H, King K. Emergency department management of mosquitoborne illness: malaria, dengue, and west nile virus. Emergency Medicine Practice. 2014;16:1-23.

3. Chen X, Gao D. Effects of travel frequency on the persistence of mosquito-borne diseases. Discrete and Continuous Dynamical Systems Series. 2020;25:4677-4701.

4. U.S. Department of health and human services: centers for disease control and prevention (CDC). Illnesses on the Rise. 2018.

5. World health organization. Yellow fever: fact sheet. 2020.

6. Lowe R, Ryan S, Mahon R, et al. Building resilience to mosquito-borne diseases in the caribbean. PLOS Biology. 2020;18:1-10.

7. Patz J, Reisen W. Immunology, climate change and vector-borne diseases. Trends in Immunology. 2001;22:171-172.

8. Morse W, Izenour K, Mckenzie B, et al. Perceptions and practices of mosquito-borne diseases in alabama - is concern where it should be? BMC Public Health, 19. 2019.

9. Franklinos L, Jones K, Redding D, et al. The effect of global change on mosquito-borne disease. The Lancet. 2019;19:E302-E312.

10. Reiter P. Climate change and mosquito-borne disease. Environmental Health Perspectives. 2001;109,141-161.

11. U.S. Wildlife service. History of bald eagle decline, protection and recovery. 2020.

12. Huang W, Wang S, Jacobs-Lorena M. Use of microbiota to fight mosquitoborne disease. Frontiers in Genetics. 11. 2020.

13. Zheng $\mathrm{X}$, Zhang D, Li Y, et al. Incompatible and sterile insect techniques combined eliminate mosquitoes. Nature. 2019;572:56-61. 